\title{
Circulating mRNA Expression of astrocyte-Elevated Gene-1 Associated with Treatment Response and Survival in Non-Small Cell Lung Cancer Patients Treated with Pemetrexed
}

\section{You-Lung Chang}

NTUH Yun-Lin Branch: National Taiwan University Hospital Yun Lin Branch

\section{Yen-Fu Chen}

NTUH Yun-Lin Branch: National Taiwan University Hospital Yun Lin Branch

\section{Ying-Yin Chen}

NTUH Yun-Lin Branch: National Taiwan University Hospital Yun Lin Branch

\section{Shih-Chieh Chang}

National Yang-Ming Medical College: National Yang-Ming University

\section{Cheng-Yu Chang}

Far Eastern Memorial Hospital

\section{Yu-Feng Wei}

Eda Hospital: E-Da Hospital

Chung-Yu Chen ( $\nabla$ c8101147@ms16.hinet.net )

National Taiwan University Hospital Yun Lin Branch https://orcid.org/0000-0001-9002-7255

\section{Research}

Keywords: lung cancer, pemetrexed, angiogenesis, oncogene, liquid biopsy, astrocyte-elevated gene-1, thymidylate synthase

Posted Date: May 7th, 2021

DOl: https://doi.org/10.21203/rs.3.rs-472588/v1

License: (9) This work is licensed under a Creative Commons Attribution 4.0 International License. Read Full License

Version of Record: A version of this preprint was published at Applied Sciences on November 30th, 2021. See the published version at https://doi.org/10.3390/app112311334. 


\section{Abstract}

Backgrounds: Astrocyte-elevated gene-1 (AEG-1) functions as an oncogene and regulates angiogenesis in non-small cell lung cancer (NSCLC). In this prospective study, we assessed the values of plasma AEG-1 mRNA expression by liquid biopsy associated with tumor response and survival in NSCLC patients treated with pemetrexed.

Methods: Patients diagnosed advanced NSCLC were enrolled to be treated with pemetrexed combined platinum as first-line chemotherapy. All patients underwent blood sampling before any cancer treatment (CO) and at first response evaluation after two cycles (C2) treatments. Response to chemotherapy and survival were assessed. Plasma mRNA was extracted from peripheral blood mononuclear cell (PBMC) and quantification of RNA was performed by real-time PCR.

Results: A total of 50 patients with advanced NSCLC were included and 13 of 50 patients combined with bevacizumab. In patient groups of SD $(n=13)$ and PD $(n=10)$, the plasma mRNA of AEG-1, thymidylate synthase (TS) and CK19 were elevated significantly at $\mathrm{C} 2$ compared to patients in treatment response group (PR, $\mathrm{n}=27$ ) (PR v.s. SD or PD, AEG-1: $1.22 \pm 0.80$ v.s. $4.51 \pm 15.45, p=0.043$ ). NSCLC patients had elevated AEG-1 (AEG-1 $\geq 2$ ) after 2-cycle chemotherapy had shorter PFS and OS (high AEG-1 v.s. low AEG-1, median, PFS: 5.5 v.s. 11.9 months, $p=0.021$; OS: 25.9 v.s. 40.8 months, $p=0.019$, respectively). In Cox regression analysis, increased plasma mRNA expression of AEG-1indicated poor prognosis in survival.

Conclusion: Circulating mRNA concentration of AEG-1 could be a predictive and prognostic biomarker in NSCLC patients treated with pemetrexed. Increased expression of AEG-1 contributed to the chemoresistance and caused lung cancer progression.

\section{Introduction}

Lung cancer is the world leading cause of death by cancer (1), and it is usually diagnosed at an advanced or metastatic stage. Treatments for advanced stages are based on cytotoxic chemotherapy and other systemic therapies (2). Response to treatment is assessed by computed tomography (CT) using the RECIST (response evaluation criteria in solid tumors) criteria (3). Although clinical image presentation is often delay to indicate cancer progression, to date, there is no reliable biomarker for the evaluation of response to standard chemotherapy treatment in lung cancer. Therefore, there is a need for more prognostic factors to manage patients with advanced non-small cell lung cancer (NSCLC).

Expression of astrocyte-elevated gene-1 (AEG-1), a novel oncoprotein, is elevated in multiple cancers and plays a vital role in tumor cell growth, invasion, angiogenesis, and progression to metastasis $(4,5)$. High expression of AEG-1 could promote the carcinogenesis and leading to a poor clinical prognosis of NSCLC (6-8). Recent findings suggest that AEG-1 contributes to broad-spectrum resistance to various chemotherapeutics $(9,10)$. Our previous data demonstrated that thymidylate synthase (TS) expression might be regulated by AEG- 1 and that increased expression of these proteins contributes to lung cancer 
disease progression and may be associated with the development of resistance to pemetrexed (11). AEG 1 could be used as a biomarker to identify subgroups of patients who require more intensive treatments. However, the acquisition of tumor tissue via biopsy may be very difficult to perform at the onset of resistance after chemotherapy since small lesions or patients' poor conditions.

Liquid biopsy analysis is currently a rapidly expanding field in translational cancer research as it might be useful at different points of the diagnostic/therapeutic course of cancer patients (12). Because messenger RNAs (mRNA) possess a critical role in intracellular protein translation and, it is likely that extracellular mRNAs reflect the status of the intracellular process, and are conceivably potential biomarkers for cancer diagnosis or therapeutic monitoring (13). Recent studies reported various coding RNAs in plasma or serum from patients with cancer, and levels of circulating cell free mRNAs were found to be predictive of clinical outcome $(14,15)$ and disease prognosis $(16,17)$.

It has been hypothesized that circulating mRNA of AEG-1 expression could be a predictive factor of tumor response and a good candidate for a prognostic factor. Liquid biopsy analysis is currently a rapidly expanding field in translational cancer research as it might be useful at different points of the diagnostic/therapeutic course of cancer patients. Some studies have shown that circulating mRNA concentration could be a predictive factor of tumor response to surgery and to radiation therapy in cancer $(18,19)$, but very few studies have investigated circulating mRNA concentration as a predictive factor of tumor response to chemotherapy (20-22). In this prospective study, we will assess the predictive and prognostic values of AEG-1, combined with TS and CK19 expression, on tumor response and survival according to circulating mRNA concentration by liquid biopsy in NSCLC patients treated with pemetrexed.

\section{Material And Methods}

\subsection{Patients and data collection}

This prospective study was approved by the investigational review boards of the National Taiwan University Hospital (NO. 201510131RINB), and all participating patients provided informed consent before chemotherapy. Patients with a diagnosis of locally advanced or metastatic non-squamous cell lung cancer, stage IIIB or IV (7th version of the TNM staging system for lung cancer of the International Association for the Study of Lung Cancer) (23), and those who were treated with pemetrexed plus platinum as first-line chemotherapy regimen in the National Taiwan University Hospital Yunlin Branch were potentially eligible for inclusion. The diagnosis of NSCLC was based on histological examination of biopsy specimens or on cytological analysis of samples aspirated with a fine needle. Molecular testing for epidermal growth factor receptor (EGFR) mutations and anaplastic lymphoma kinase (ALK) rearrangements were investigated from the pathologic or cytological specimens. Patients' basic data and medication usage were retrieved from electronic medical records.

\subsection{Evaluation of treatment responses and survival}


The primary objective was to identify associations between specific biomarkers and treatment response rate. The secondary objective was to identify biomarkers associated with patient survival. All patients underwent blood sampling before chemotherapy (C0) and after 2 cycles of chemotherapy (C2).

The patients were administered with cisplatin or carboplatin plus pemetrexed intravenously every three weeks in patients with wild-type EGFR and ALK mutation or patients harboring EGFR or ALK mutation with disease progression after target therapy. Furthermore, patients received $500 \mathrm{mg} / \mathrm{m} 2$ pemetrexed intravenously every three weeks as continuation maintenance therapy if the disease did not progress after 4 cycles of the first-line treatment. All patients were adults with good performance status (Eastern Cooperative Oncology Group Performance Status: 0-1). Treatment was discontinued if there was evidence of progressive disease, or unacceptable toxicity.

Chest computed tomography was performed every 3 months as routine clinical practices, and as needed to confirm treatment response. Treatment responses were evaluated according to the Response Evaluation Criteria in Solid Tumors, version 1.1 (24), and were defined as complete remission, partial response, stable disease, and progressive disease. Response rate (RR) was defined as the percentage of patients who achieved a complete remission or partial response. Disease-control rate (DCR) was defined as the percentage of patients who achieved a complete remission, partial response, or stable disease.

Progression-free survival (PFS) was defined as the time from the first cycle of pemetrexed plus platinum to documented progression or death from any cause, and was censored at the date of the last follow-up visit for patients who were still alive and who had not exhibited progression. The overall survival (OS) was measured from the date of the start of the treatment to the date of the last follow-up. Patients were followed up until December 2018.

\subsection{Isolation of Human PBMCs}

Collect $4 \mathrm{ml}$ of human venous blood sample in heparinised vials (BD biosciences) and mix well by gently inverting the tube several times. Take $4 \mathrm{ml}$ of Ficoll Histopaque in a $15 \mathrm{ml}$ centrifuge tube. Gently layer the blood on the top of Ficoll Histopaque. The layering should be done very slowly that blood and Ficoll Histopaque should stay as two different layers. Centrifuge the tubes (without any delay) for $\mathbf{3 0}$ min at $1200 \mathrm{~g}$ in $4^{\circ} \mathrm{C}$ in a swing-out bucket. Fixed angle rotors also can be used but would require more caution when separating cells in interphase. Aspirate the whitish buffy coat (about $1 \mathrm{ml}$ ) (PBMCs) formed in the interphase between histopaque and medium. Wash the cells (centrifuge in $1000 \mathrm{~g}$ for $10 \mathrm{~min}$ ) twice with $10 \mathrm{ml}$ of sterile PBS or sterile Dulbecco's modified eagle medium. The approximate yield of cells added 1 $\mathrm{ml}$ Trizol and placed in the $-80^{\circ} \mathrm{C}$ refrigerator for freezing.

\subsection{Plasma mRNA Extraction and Quantitative Real-time PCR.}

Total RNA (from PBMCs) was extracted using a QIAamp RNA Blood Mini Kit (Qiagen). First-strand complementary DNA (cDNA) synthesis was performed using $2 \mu \mathrm{g}$ of total RNA using Invitrogen Super Script Reverse Transcriptase (Thermo Fisher Scientific, Carlsbad, CA, USA). Real-time PCR was performed 
according to the manufacturer's protocol for Applied Biosystems Power SYBR® Green PCR Master Mix, and the relative mRNA expression level of AEG-1(Astrocyte elevated gene-1, Metadherin, MTDH), TS (Thymidylate synthetase, TYMS), CK19 (Keratin19) and Actin was determined as a ratio to the actin.

\subsection{Statistical methods}

Variables as plasma mRNA level including AEG-1, TS and CK19 were presented as mean \pm SD. The mRNA expression level before chemotherapy was indicated as baseline as 1.0. For comparisons of these biomarker variables, we used the independent T-test and paired T-test, respectively. Categorical variables are expressed as percentages. The median value of plasma AGE-1, TS, and CK19 mRNA expression was captured as cut-off level. Correlation of patients' clinical fator and treatment response was estimated by using the Pearson Chi-Square. For survival analysis we used the Kaplan-Meier method and median survival (and corresponding 95\% confidence intervals) were compared using the log-rank test. Then, we used a Cox regression model (enter method) in order to assess variables associated with survival. If $p<$ 0.05 was considered significant. Statistical analysis was performed using MedCalc (Version 19.6.1).

\section{Results}

\subsection{Clinical Evaluation of Treatment Efficacy}

From the year 2015 to 2018, a total of 50 patients (29 male and 21 female) with advanced nonsquamous NSCLC received Pemetrexed/Platinum based doublets chemotherapy. The median of age was 65 years old (range: 37 to 92 years old). Twenty-two of them harbored EGFR mutation and three of them were ALK positive. Chemotherapy regimens included 36 cisplatin and 14 carboplatin with pemetrexed, thirteen of 50 patients combined with bevacizumab (Table 1). 
Table 1

Pemetrexed plus Platinum with and without Bevacizumab for NSCLC: Basic Characteristics and Treatment Response

\section{Overall \\ $P C(n=37) \quad$ PCB $(n=13)$}

Age, years old, (median, range)

$65(37-92)$

$68(37-92)$

$62(47-76)$

\section{Gender}

Male

$29(58.0 \%)$

$25(67.6 \%)$

$4(30.8 \%)$

Female

$21(42.0 \%)$

$12(32.4 \%)$

$9(69.2 \%)$

\section{Smoking status}

Current or ever

$21(42.0 \%)$

$18(48.6 \%)$

$3(23.1 \%)$

Never

$29(58.0 \%)$

19 (51.4\%)

$10(76.9 \%)$

Mutation

EGFR

ALK

Wild type

Platinum

Cisplatin

Carboplatin

\section{Treatment Response}

PR

SD

PD

\section{Response rate (\%)}

Disease control rate (\%)
$22(44.0 \%)$

$3(6.0 \%)$

$25(50.0 \%)$

$36(62.0 \%)$

$14(28.0 \%)$

$26(52.0 \%)$

$18(48.6 \%)$

$10(27.0 \%)$

$9(24.3 \%)$

$48.6 \%$

$75.7 \%$

$10(20.0 \%)$

$52 \%$

$80 \%$
$6(46.2 \%)$

$1(7.6 \%)$

$6(46.2 \%)$

$8(61.5 \%)$

$5(38.5 \%)$

$14(24.0 \%) \quad 10(27.0 \%) \quad 4(30.8 \%)$

NSCLC: non-small cell lung cancer; PC: pemetrexed/platinum; PCB: pemetrexed/platinum/bevacizumab; EGFR: epidermal growth factor receptor; ALK: anaplastic lymphoma kinase; PR: partial response; SD: stable disease; PD: progressive disease

The initial response rate of chemotherapy included 26 partial responses, 12 stable diseases and 10 progressive diseases. The treatment response rate and disease-control rate of Pemetrexed/Platinum doublet chemotherapy were $48.6 \%$ (18/37) and 75.7\% (28/37), respectively. The treatment response rate and disease-control rate of bevacizumab-combined chemotherapy were $61.5 \%(8 / 13)$ and $92.3 \%(12 / 13)$, respectively (Table 1). There was no significant difference of treatment response rate (RR) and disease- 
control rate (DCR) between Pemetrexed/Platinum doublet (PC) and bevacizumab - combined chemotherapy (PCB) (RR: $p=0.200$ and DCR: $p=0.197$, respectively). Patients treated with bevacizumab combined chemotherapy had significant longer progression-free survival (PFS) and overall survival (OS) (PCB v.s. PC, PFS, median, 15.4 v.s. 7.7 months, $p=0.01$; OS, median, 40.8 v.s. 28.9 , months, $p=0.02$ ) (Fig. 1).

\subsection{The Association of Plasma Biomarks and Clinical Treatment Response}

Plasma biomarks including AEG-1, thymidylate synthase (TS) and CK19 were analyzed before chemotherapy (C0) and after 2 cycles chemotherapy (C2). In patient groups of SD ( $n=14)$ and PD ( $n=$ 10), the plasma mRNA of AEG1, TS and CK19 were elevated significantly at C2 compared to patients in treatment response group (PR, $\mathrm{n}=26$ ) (PR v.s. $\mathrm{SD}$ or $\mathrm{PD}, \mathrm{AEG}-1: 1.22 \pm 0.80$ v.s. $4.51 \pm 15.45, p=0.043$; TS: $1.76 \pm 1.77$ v.s. $10.22 \pm 23.95, p=0.003$; CK9: $3.05 \pm 7.86$ v.s. $245.03 \pm 884.14, p=0.005$, respectively) (Fig. 2). Plasma AEG-1 mRNA expression was lower in patients treated with bevacizumab at $\mathrm{C} 2$ but not significantly (with bevacizumab v.s. without, AEG-1: $1.25 \pm 0.75$ v.s. $3.25 \pm 12.20, p=0.560$ ). Moreover, the change of plasma mRNA level as AEG-1, TS and CK19 between different chemotherapy regimens including either cisplatin or carboplatin was not significant.

\subsection{The Elevation of Circulating AEG-1 mRNA Expression Indicated Poor Prognosis}

NSCLC patients with elevated plasma AEG-1 mRNA expression (AEG-1 $\geq 2$ ) after 2-cycle chemotherapy had significant shorter PFS (high AEG-1 v.s. low AEG-1, PFS, median, 5.5 v.s. 11.9 months, $p=0.021$ ). Otherwise, elevated plasma TS ( TS $\geq 5$ ) and CK19 (CK19 $\geq 50)$ mRNA expression after 2-cycle chemotherapy also had shorter PFS (High TS v.s. low TS, PFS, median, 5.8 v.s. 11.9 months, $p=0.501$; high CK19 v.s. low CK19, PFS, median, 5.5 v.s. 11.9 months, $p=0.474$; respectively) (Fig. 3).

NSCLC patients with elevated plasma AEG-1 mRNA expression ( $A E G-1 \geq 2$ ) after 2-cycle chemotherapy leaded to poor overall survival (high AEG-1 v.s. Iow AEG-1, OS, median, 25.9 v.s. 40.8 months, $p=0.019$ ). Elevated plasma TS ( TS $\geq 5$ ) and CK19 ( CK19 $\geq 50$ ) mRNA expression after 2-cycle chemotherapy also had poor overall survival (High TS v.s. Iow TS, OS, median, 34.0 v.s. 59.0 months, $p=0.853$; high CK19 v.s. Iow CK19, OS, median, 29.3 v.s. 34.0 months, $p=0.880$; respectively) (Fig. 4).

In Cox regression multivariant analysis, increased circulating mRNA expression of AEG-1 can be a poor prognostic mark in survival (Hazard ratio [HR]: 9.68, 95\% Cl: $1.69-55.57, p=0.01$ ), but not TS and CK19 (Fig. 5).

\section{Discussion}


In our previous study, AEG-1 was conferred to induce resistance to pemetrexed in non-small cell lung cancer by upregulating TS expression in vivo and in vitro [9]. Here, we reported that elevated circulating mRNA expression of AEG-1 and TS negatively affects treatment response and contributes to the poor prognosis in NSCLC patients treated with pemetrexed. Our data indicated a significant correlation between AEG-1 and TS plasam mRNA expression levels and treatment efficacy in NSCLC. Furthermore, we demonstrated that circulating AEG-1 mRNA expression detected by liquid biopsy after chemotherapy were associated with disease prognosis and survival.

Circulating tumor cells (CTCs) are crucial to tumor metastasis and valuable for prediction of clinical outcome in patients with NSCLC using "liquid biopsies". $(25,26)$. Chemotherapy is a standard mode of treatment for all cancers. CTC levels are determined before and after rounds of chemotherapy. $(27,28)$ However, isolation and enumeration of CTCs is difficult because of their low numbers, size, and heterogeneity. Novel techniques for CTCs capture and detection from peripheral blood mononuclear cells (PBMCs) had been developed $(29,30)$, and CTCs were counted $0.01-0.04 \%$ of total PBMCs (31). Our study indicated that the change of AEG-1, TS and CK19 plasma mRNA expression after chemotherapy were associated with disease prognosis and survival detected by RT-PCR from PBMC. Therefore, analysis of gene expression in PBMC could be used potentially to replace the techniques to capture CTC.

Many studies have shown that TS expression level could be suggestive of the objective response of patients with NSCLC treated with pemetrexed containing chemotherapy (32-39). Our previous study revealed that increased expression of AEG-1 induces TS expression and contributes to the resistance of pemetrexed in cultured cells and in a few rebiopsy cases by IHC stain (9). In this study, we investigated the plasma AEG-1 and TS mRNA expression by RT-PCR to detect that increased expression of AEG-1 and TS induces poor response to pemetrexed-contained chemotherapy. Furthermore, our analysis demonstrated about the negative correlation between AEG-1 and TS expression levels and prognosis in advanced NSCLC patients treated with pemetrexed.

CK19 is expressed in NSCLC and is a prognostic determinant in lung cancer (40-42). Bastawisy AE et al. (43) demonstrated that high CK19 was found with prognostic value in cases showing progression disease. However, there was no statistically significant correlation between high CK19 and overall survival. This is in contrary to the results reported by Bréchot et al. (44) and Rosenblatt et al. (45) who showed significant correlation with survival. In our study, plasma CK19 mRNA expression were elevated significantly in patients with poor treatment response. Though, the role of CK19 expression was not significant in survival. This may be explained by the small sample size of the present study.

AEG-1 was confirmed to have functions as an oncogene and regulates angiogenesis $(4,5)$. AEG-1 expression directly correlates with increased expression of angiogenesis markers including angiopoietin-1 (Ang1), matrix metalloprotease (MMP)-2, and HIF1- $a$ and supporting a potential role of AEG-1 in tumor angiogenesis (46). AEG-1 mRNA expression upregulation indicated a significantly positive correlation with vascular endothelial growth factor (VEGF) expression and increased intratumoral microvessel density (iMVD, labeled by CD105) counting (47). Several studies have showed that over-expression of 
AEG-1 significantly associates with tumor aggressiveness and poor prognosis in $\operatorname{NSCLC}(48,49)$. These results suggested that AEG-1 may play important roles in malignant transformation and tumor angiogenesis in NSCLC, and anti-AEG-1 mRNA expression may be a novel potential strategy for antiangiogenic therapy of NSCLC. In our study, bevacizumab-combined chemotherapy decreased more plasma AEG-1 mRNA expression, also had higher response rate, longer PFS and OS. However, the differences were not significantly. This may be limited by the small sample size of the present study.

\section{Conclusion}

AEG-1 functions as an oncogene and regulates angiogenesis in NSCLC. AEG-1 also promotes TS expression contributing to the development of resistance to pemetrexed. Bevacizumab-combined chemotherapy inhibits AEG-1 expression and could increase treatment benefits and survivals. Plasma AEG-1 mRNA level could be used a predictive and prognostic biomarker in NSCLC patients treated with pemetrexed. Increased expression of AEG-1 leaded to chemoresistance and also caused lung cancer progression, which could be used for clinical evaluation combined with image studies.

\section{Declarations}

\section{Ethics approval and consent to participate}

This study was approved by the Institutional Review Board of National Taiwan University Hospital Yunlin Branch (No. 201510131RINB). Patients with lung cancer provided written informed consent for the use of blood specimens and clinical data for research prior to undergoing chemotherapy.

\section{Consent for publication}

\section{Availability of supporting data}

The authors confirm that the data supporting the findings of this study are available within the article.

\section{Competing interests}

The authors declare that they have no conflict of interest.

\section{Funding}

This study was supported by Grants NTUHYL105.X007 from the National Taiwan University Hospital Yunlin Branch for the research and laboratory works.

\section{Authors' contributions}

Conception: Chen YY, Chen CY

Interpretation or analysis of data: Change YL, Chen YF, Chen YY 
Preparation of the manuscript: Chang YL, Chen YF, Chen YY

Revision for important intellectual content: Chang SC, Chang CY

Supervision: Wei YF, Chen CY

\section{Acknowledgements}

We are indebted to the First Common Laboratory of National Taiwan University Hospital Yunlin Branch, for technical support.

\section{References}

1. Ferlay J, Soerjomataram I, Dikshit R, et al. Cancer incidence and mortality worldwide: sources, methods and major patterns in GLOBOCAN 2012. Int J Cancer 2015; 136: E359-E386.

2. Peters S, Adjei AA, Gridelli C, et al. Metastatic non-small-cell lung cancer (NSCLC): ESMO Clinical Practice Guidelines for diagnosis, treatment and follow-up. Ann Oncol 2012; 23: Suppl. 7, 56-64.

3. Eisenhauer EA, Therasse P, Bogaerts $\mathrm{J}$, et al. New response evaluation criteria in solid tumours: revised RECIST guideline (version 1.1). Eur J Cancer 2009; 45: 228-247.

4. Emdad L, Sarkar D, Su ZZ, et al. Astrocyte elevated gene-1: recent insights into a novel gene involved in tumor progression, metastasis and neurodegeneration. Pharmacol Ther. 2007;114(2):155-170.

5. Hu G, Wei Y, Kang Y. The multifaceted role of MTDH/AEG-1 in cancer progression. Clin Cancer Res. 2009;15(18):5615-20.

6. Song L, Li W, Zhang H, et al. Over-expression of AEG-1 significantly associates with tumour aggressiveness and poor prognosis in human non-small cell lung cancer. J Pathol. 2009;219(3):317326.

7. Ke ZF, Mao X, Zeng C, et al. AEG-1 expression characteristics in human non-small cell lung cancer and its relationship with apoptosis. Med Oncol. 2013;30(1):383.

8. Zhang Y, Li ZY, Hou XX, Wang X, Luo YH, Ying YP, Chen G. Clinical significance and effect of AEG-1 on the proliferation, invasion, and migration of NSCLC: a study based on immunohistochemistry, TCGA, bioinformatics, in vitro and in vivo verification. Oncotarget. 2017;8(10): 16531-16552.

9. Yoo BK, Chen D, Su ZZ, et al. Molecular mechanism of chemoresistance by Astrocyte Elevated Gene1 (AEG-1). Cancer Res. 2010;70(8):3249-3258.

10. Meng X, Thiel KW, LeslieKK. Drug resistance mediated by AEG-1/MTDH/LYRIC. Adv Cancer Res. 2013;120:135-157.

11. Chen CY, Chen YY, Chen JJW, Chen KY, Ho CC, Shih JY, Chang YL, Yu CJ, Yang PC. Astrocyte-elevated gene-1 confers resistance to pemetrexed in non-small cell lung cancer by upregulating thymidylate synthase expression. Oncotarget. 2017; 8(37): 61901-61916.

12. Alix-Panabieres, K. Pantel, Circulating tumor cells: liquid biopsy of cancer. Clin. Chem. 2013; 59 (1): 110-118. 
13. Rapisuwon S, Vietsch EE, Wellstein A. Circulating biomarkers to monitor cancer progression and treatment. Comput Struct Biotechnol J. 2016 Jun 1;14:211-22.

14. Garcia V., Garcia J.M., Pena C., Silva J., Dominguez G. Free circulating mRNA in plasma from breast cancer patients and clinical outcome. Cancer Lett. 2008;263:312-320.

15. Garcia V., Garcia J.M., Silva J., Martin P., Pena C. Extracellular tumor-related mRNA in plasma of lymphoma patients and survival implications. PLoS One. 2009;4:e8173.

16. March-Villalba J.A., Martinez-Jabaloyas J.M., Herrero M.J., Santamaria J., Alino S.F. Cell-free circulating plasma hTERT mRNA is a useful marker for prostate cancer diagnosis and is associated with poor prognosis tumor characteristics. PLoS One. 2012;7:e43470.

17. Silva J., Garcia V., Garcia J.M., Pena C., Dominguez G. Circulating Bmi-1 mRNA as a possible prognostic factor for advanced breast cancer patients. Breast Cancer Res. 2007;9:R55.

18. Zaporozhchenko IA, Ponomaryova AA, Rykova EY, Laktionov PP. The potential of circulating cell-free RNA as a cancer biomarker: challenges and opportunities. Expert Rev Mol Diagn. 2018 Feb;18(2):133-145.

19. Levy B, Hu ZI, Cordova KN, Close S, Lee K, Becker D. Clinical Utility of Liquid Diagnostic Platforms in Non-Small Cell Lung Cancer. Oncologist. 2016 Sep;21(9):1121-30.

20. Rosell R, Cecere F, Santarpia M, Reguart N, Taron M. Predicting the outcome of chemotherapy for lung cancer. Curr Opin Pharmacol. 2006 Aug;6(4):323-31

21. Du YJ, Li J, Zhu WF, Wu Y, Tang XP, Wang Y, Hu YM. Survivin mRNA-circulating tumor cells predict treatment efficacy of chemotherapy and survival for advanced non-small cell lung cancer patients. Tumour Biol. 2014 May;35(5):4499-507.

22. Gumireddy K, Li A, Chang DH, et al. AKAP4 is a circulating biomarker for non-small cell lung cancer. Oncotarget 2015;6:17637-17647.

23. Edge SB, Byrd DR, Compton CC, Fritz AG, Greene F, Trotti A (2010). AJCC cancer staging manual, 7th edition. New York, NY: Springer

24. Gwyther S, Mooney M, Rubinstein L, Schwartz LH, Sargent D, Ford R, et al (2009). New response evaluation criteria in solid tumours: revised RECIST guideline (version 1.1). Eur J Cancer 45: 228-247.

25. Levy B, Hu ZI, Cordova KN, Close S, Lee K, Becker D. Clinical Utility of Liquid Diagnostic Platforms in Non-Small Cell Lung Cancer. Oncologist. 2016;21(9):1121-30.

26. Molina-Vila MA, Mayo-de-Las-Casas C, Giménez-Capitán A, Jordana-Ariza N, Garzón M, Balada A, Villatoro S, Teixidó C, García-Peláez B, Aguado C, Catalán MJ, Campos R, Pérez-Rosado A, BertranAlamillo J, Martínez-Bueno A, Gil MD, González-Cao M, González X, Morales-Espinosa D, Viteri S, Karachaliou N, Rosell R. Liquid Biopsy in Non-Small Cell Lung Cancer. Front Med (Lausanne). 2016;3:69.

27. Coco S, Alama A, Vanni I, Fontana V, Genova C, Dal Bello MG, Truini A, Rijavec E, Biello F, Sini C, Burrafato G, Maggioni C, Barletta G, Grossi F. Circulating Cell-Free DNA and Circulating Tumor Cells as Prognostic and Predictive Biomarkers in Advanced Non-Small Cell Lung Cancer Patients Treated with First-Line Chemotherapy. Int J Mol Sci. 2017 May 11;18(5). pii: E1035. 
28. Zhou J, Dong F, Cui F, Xu R, Tang X. The role of circulating tumor cells in evaluation of prognosis and treatment response in advanced non-small-cell lung cancer. Cancer Chemother Pharmacol. 2017 Apr;79(4):825-833

29. Li H, Meng QH, Noh H, Batth IS, Somaiah N, Torres KE, Xia X, Wang R, Li S. Detection of circulating tumor cells from cryopreserved human sarcoma peripheral blood mononuclear cells. Cancer Lett. 2017:403:216-223.

30. Wang F, Li YC, Liu LP, Zhang HM, Tong S. Circulating Tumor Cells and Tumor Stem Cells Detection in the Peripheral Blood Mononuclear Cells of Breast Cancer. J Clin Lab Anal. 2016 Sep;30(5):616-22.

31. Pravin D. Potdar, Keerti Sen. Profiling of circulating tumor cells in liquid biopsies from metastatic cancer patients. J Cancer Metastasis Treat 2017;3:6-15

32. Liu Y, Yin TJ, Zhou R, Zhou S, Fan L, Zhang RG. Expression of thymidylate synthase predicts clinical outcomes of pemetrexed-containing chemotherapy for non-small-cell lung cancer: a systemic review and meta-analysis. Cancer Chemother Pharmacol. 2013; 72:1125-1132.

33. Wang T, Chuan Pan C, Rui Yu J, Long Y, Hong Cai X, De Yin X, Qiong Hao L, Li Luo L. Association between TYMS expression and efficacy of pemetrexed-based chemotherapy in advanced non-small cell lung cancer: a meta-analysis. PLoS One. 2013; 8:e74284.

34. Nicolson MC, Fennell DA, Ferry D, O'Byrne K, Shah R, Potter V, Skailes G, Upadhyay S, Taylor P, André V, Nguyen TS, Myrand SP, Visseren-Grul C, et al. Thymidylate synthase expression and outcome of patients receiving pemetrexed for advanced nonsquamous non-small-cell lung cancer in a prospective blinded assessment phase II clinical trial. J Thorac Oncol. 2013; 8:930-939.

35. Wang L, Wang R, Pan Y, Sun Y, Zhang J, Chen H. The pemetrexed-containing treatments in the nonsmall cell lung cancer is -/low thymidylate synthase expression better than $+/$ high thymidylate synthase expression: a meta-analysis. BMC Cancer. 2014; 14:205.

36. Christoph DC, Asuncion BR, Hassan B, Tran C, Maltzman JD, O'Shannessy DJ, Wynes MW, Gauler TC, Wohlschlaeger J, Hoiczyk M, Schuler M, Eberhardt WE, Hirsch FR, et al. Significance of folate receptor alpha and thymidylate synthase protein expression in patients with non-small-cell lung cancer treated with pemetrexed. J Thorac Oncol. 2013; 8:19-30.

37. Chamizo C, Zazo S, Dómine M, Cristóbal I, García-Foncillas J, Rojo F, Madoz-Gúrpide J. Thymidylate synthase expression as a predictive biomarker of pemetrexed sensitivity in advanced non-small cell lung cancer. BMC Pulm Med. 2015; 15:132.

38. Kasai D, Ozasa H, Oguri T, Miyazaki M, Uemura T, Takakuwa O, Kunii E, Ohkubo H, Maeno K, Niimi A. Thymidylate synthase gene copy number as a predictive marker for response to pemetrexed treatment of lung adenocarcinoma. Anticancer Res. 2013; 33:1935-1940.

39. Wang X, Wang Y, Wang Y, Cheng J, Wang Y, Ha M. Association of thymidylate synthase gene 3' untranslated region polymorphism with sensitivity of non-small cell lung cancer to pemetrexed treatment: TS gene polymorphism and pemetrexed sensitivity in NSCLC. J Biomed Sci. 2013; 20:5.

40. Pujol, JL, Molinier, O, Ebert, W, Daures, JP, Barlesi, F, Buccheri, G, Paesmans, M, Quoix, E, Moro-Sibilot, D, Szturmowicz, M, Brechot, JM, Muley, T, Grenier, J. CYFRA 21-1 is a prognostic determinant in non- 
small-cell lung cancer: results of a meta-analysis in 2063 patients. $\mathrm{Br} \mathrm{J}$ Cancer 2004; 90: 2097- 105

41. Yan L, Yao Y, Wang LH, Wang ML, Fu XH. Detection of CK19, LUNX, and KS1/4 mRNA expression in the peripheral blood for diagnosis of micrometastases in patients with non-small cell lung cancer and their clinical implications. 2015 Nov 25;14(4):15090-5.

42. Prognostic value of chemotherapy-resistant CK19 mRNA-positive circulating tumor cells in patients with advanced/metastatic non-small cell lung cancer. Milaki G, Messaritakis I, Koinis F, Kotsakis A, Apostolaki S, Dermitzaki EK, Perraki M, Hatzidaki D, Georgoulias V. Cancer Chemother Pharmacol. 2017 Jul;80(1):101-108.

43. Bastawisy AE, Azzouny ME, Mohammed G, Allah AA, Behiry E. Serum cytokeratin 19 fragment in advanced lung cancer: could we eventually have a serum tumor marker? 2014 Jan 30;8:394.

44. M. Bréchot et al (2002) Diagnostic and prognostic value of Cyfra 21-1 compared with other tumour markers in patients with non-small cell lung cancer: a prospective study of 116 patients Service de Pneumologie, Département de Biostatistique et Informatique Médicale, Hôpital Saint-Louis, France Available online 22 July 2002.

45. Rosenblatt PY et al (2009) Lung cancer (NSCLC): CALGB 150304 Proc. 2009 ASCO Annual Meeting Session Type and Session Title: Poster Discussion Session, Tumour Biology and Human Genetics Abstract No:11020 J Clin Oncol 27 15s.

46. Emdad L, Lee SG, Su ZZ, Jeon HY, Boukerche H, Sarkar D, Fisher PB. Astrocyte elevated gene-1 (AEG1) functions as an oncogene and regulates angiogenesis. Proc Natl Acad Sci U S A. 2009 Dec 15;106(50):21300-5.

47. Ma Z, Chen Y, Dong S, Xu X, Liu J, Song P, Yu C, Dai L. AEG-1 mRNA expression in non-small cell lung cancer is associated with increased tumor angiogenesis. Pathol Res Pract. 2017 Oct;213(10):12571263.

48. Song L1, Li W, Zhang H, Liao W, Dai T, Yu C, Ding X, Zhang L, Li J. Over-expression of AEG-1 significantly associates with tumour aggressiveness and poor prognosis in human non-small cell lung cancer. J Pathol. 2009 Nov;219(3):317-26.

49. Ding Q, Chen Y, Dong S, Xu X, Liu J, Song P, Yu C, Ma Z. Astrocyte elevated gene-1 is overexpressed in non-small-cell lung cancer and associated with increased tumour angiogenesis. Interact Cardiovasc Thorac Surg. 2018 Mar 1;26(3):395-401.

\section{Figures}



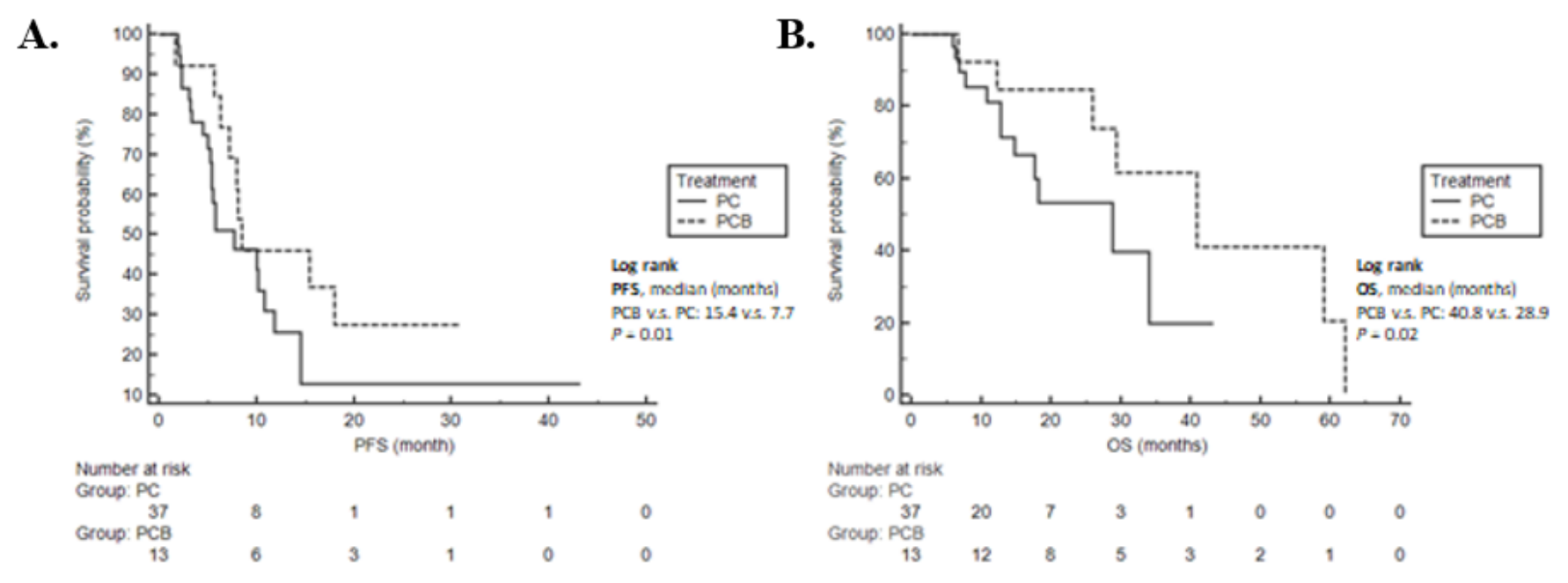

\section{Figure 1}

Kaplan-Meier survival analysis illustrated chemotherapy combination affects (A) progression-free survival (PFS) and (B) overall survival (OS). Non-small cell lung cancer (NSCLC) patients treated with bevacizumab - combined pemetrexed/platinum doublet chemotherapy (PCB) had significant longer PFS and OS compared to pemetrexed/platinum doublet (PC) (PCB v.s. PC, PFS, median, 15.4 v.s. 7.7 months, $p=0.01 ;$ OS, median, 40.8 v.s. 28.9 , months, $p=0.02$. 
A.

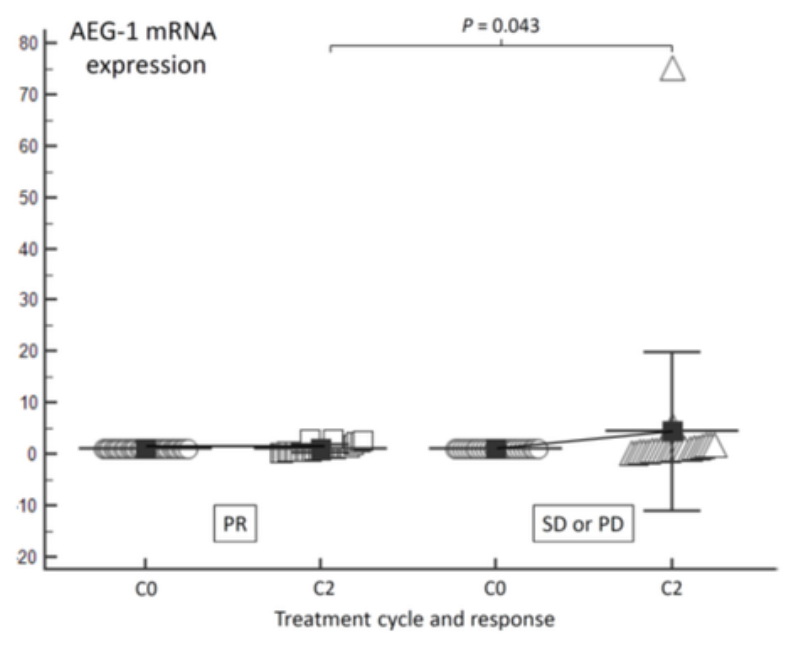

B.

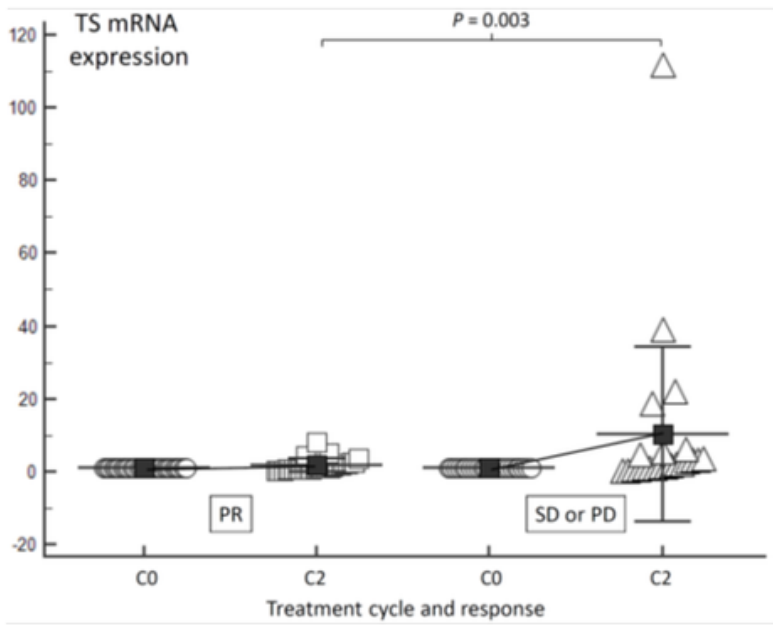

C.

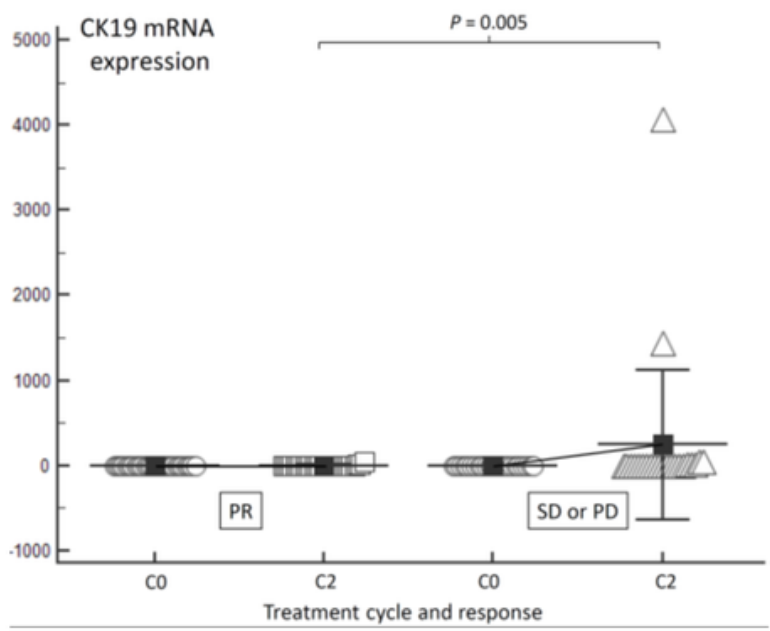

\section{Figure 2}

Dot plots with mean \pm standard deviation demonstrated plasma mRNA expression change of $(A)$ astrocyte-elevated gene-1 (AEG-1), (B) thymidylate synthase (TS) and (C) CK19 in non-small cell lung cancer (NSCLC) patients before chemotherapy (C0) and after 2 cycles chemotherapy (C2). Patients with stable disease (SD) or progression disease (PD) had significant increased plasma mRNA expression of AEG-1, TS and CK19, compared to those with partial response (PR) after 2 cycles chemotherapy. 

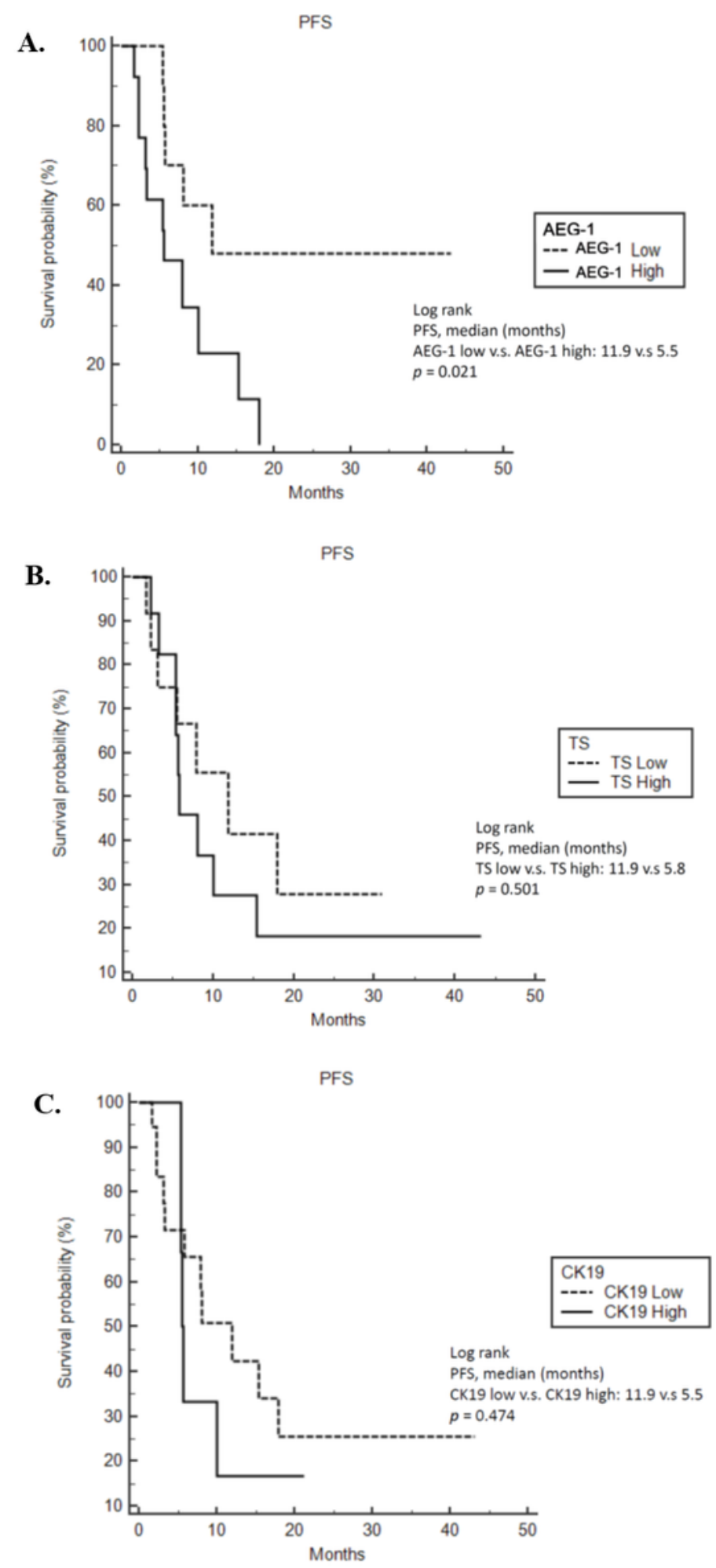

\section{Figure 3}

Expression level of plasma biomarker affects progression-free survival (PFS) plots by Kaplan-Meier survival analysis. (A) Patients with low and high levels of astrocyte-elevated gene-1 (AEG-1) expression are illustrated. PFS for patients with low AEG-1 expression was significantly longer (low vs. high, 11.9 v.s. 5.5 months, $p=0.02$, log rank test). (B) Patients with low and high levels of thymidylate synthase (TS) and, (C) CK19. 

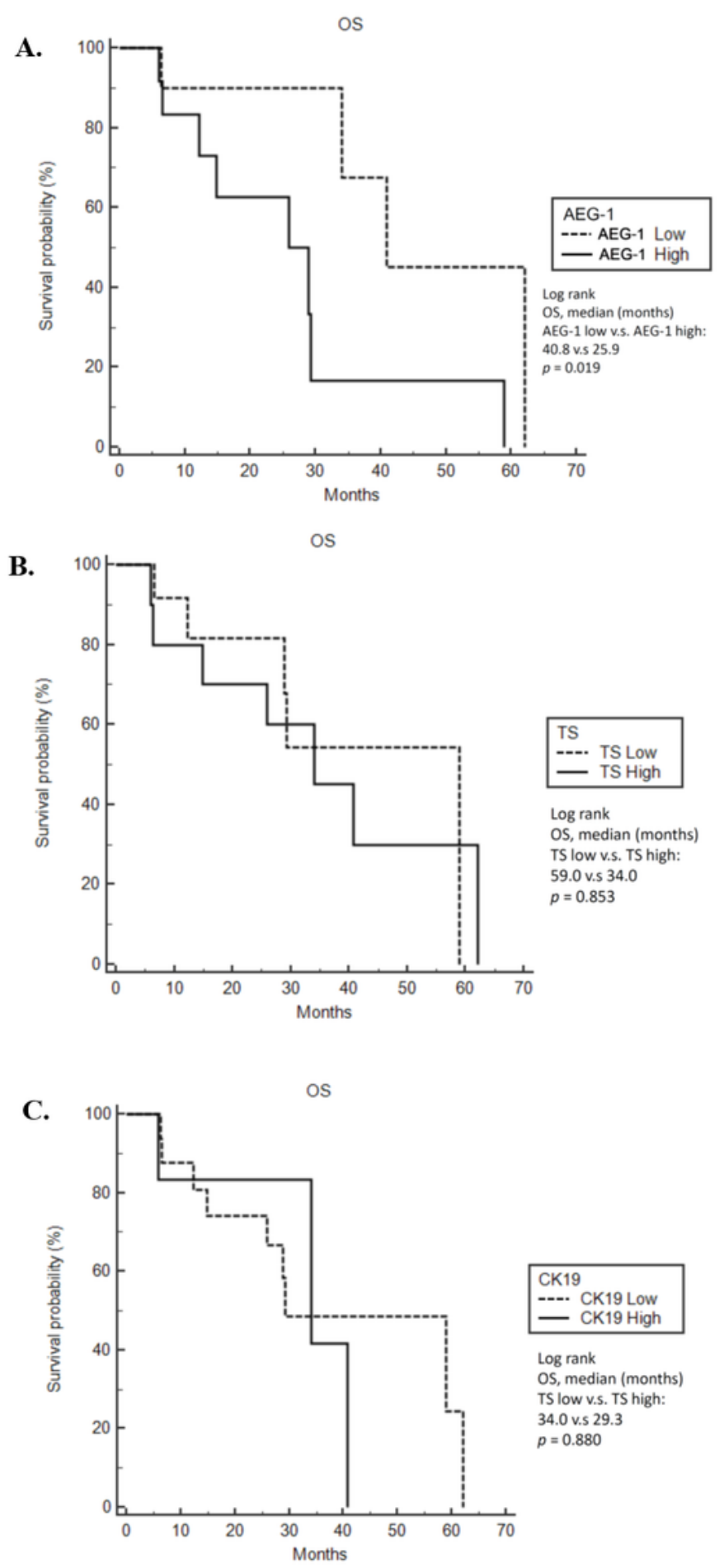

\section{Figure 4}

Overall survival (OS) for patients with expression level of plasma biomarker plots by Kaplan-Meier survival analysis. (A) OS for patients with low AEG-1 expression was significantly longer (low vs. high, 40.8 vs. 25.9 months, $p=0.019$, log rank test). (B) Patients with low and high levels of thymidylate synthase (TS) and, (C) CK19. 


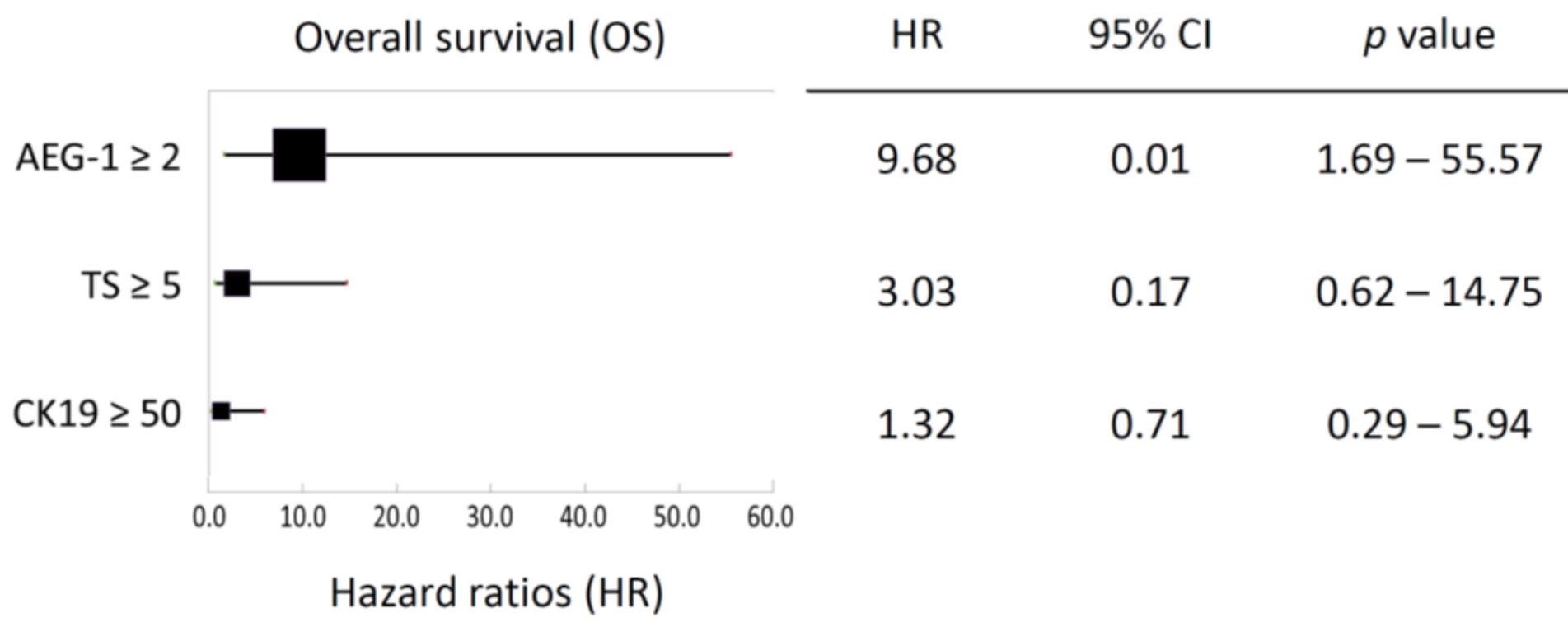

Figure 5

Cox regression multivariate analysis associated with overall survival (OS). AEG1: astrocyte-elevated gene$1 ;$ TS: thymidylate synthase 